\title{
Knowledge, attitudes and self-care practices of patients with glaucoma in uThungulu in KwaZulu-Natal
}

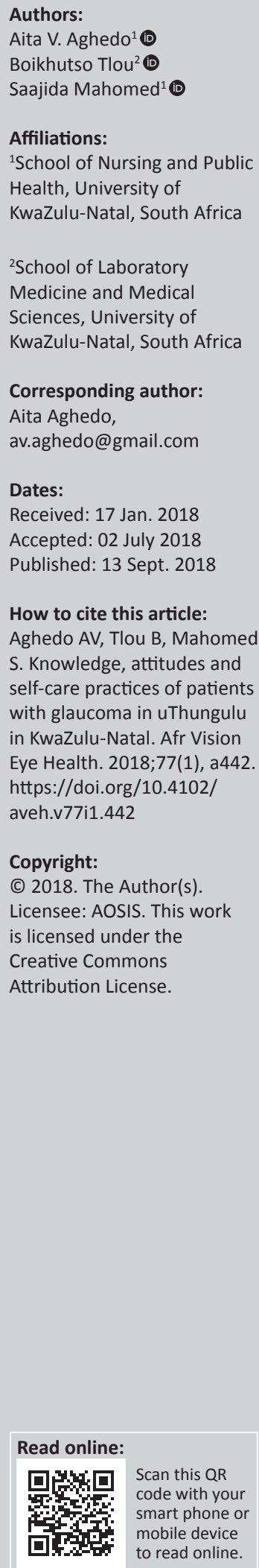

Background: Glaucoma remains one of the leading causes of blindness in South Africa. Early detection, effective treatment and strict compliance with treatment are instrumental to prevent further damage to the optic nerve and thus preserve vision.

Aim: The purpose of this study was to assess the knowledge, attitude and self-care practices of patients with glaucoma in KwaZulu-Natal.

Setting: The study was conducted in the ophthalmology outpatient department of a public hospital and a private ophthalmology practice in the uThungulu district.

Methods: This was an observational, analytic, cross-sectional study. Structured questionnaires assessing knowledge, attitudes and self-care practices were administered to patients presenting for follow-up glaucoma management.

Results: The median age of the 384 patients in this study was 60 (interquartile range [IQR] 48.5-69.5) and 59 years (IQR 49.0-66.5) for males and females, respectively ( $p>0.05)$. Two hundred and thirty-eight (62.0\%) patients had good knowledge of glaucoma. Age, gender and duration of glaucoma diagnosis were significantly associated with good knowledge of glaucoma. Three hundred and forty-four (89.6\%) patients reported having good self-care practices. Patients over the age of 65 years were significantly less likely to have good self-care practices compared to patients aged $26-45$ years (odds ratio [OR]: 0.2 , confidence interval [CI]: $0.1-0.6, p=0.01)$. Good knowledge of glaucoma was significantly associated with good selfcare practices of glaucoma $(p<0.001)$.

Conclusion: Elderly patients do not have sufficient knowledge of glaucoma and have poor self-care glaucoma practices. It is important to involve family members of these patients in the education and counselling of glaucoma to facilitate improved disease management.

\section{Introduction}

The term 'glaucoma' is used to describe a group of optic nerve disorders that if left uncontrolled result in visual field defects and associated vision loss. ${ }^{1,2}$ Glaucoma involves the death of retinal ganglion cells, which the clinician identifies by optic disc examination, visual field testing and retinal nerve fibre layer assessment with, for example, optical coherence tomography. ${ }^{3}$ Globally, glaucoma is the second commonest cause of blindness. ${ }^{3}$ An estimated 6.5 million people have glaucoma in sub-Saharan Africa. ${ }^{4}$ The prevalence of glaucoma in South Africa is approximately $5 \%$ and glaucoma contributes approximately $23 \%$ of blindness nationally. $5,6,7$

Africans are predominantly affected by primary open angle glaucoma (POAG). ${ }^{8}$ In South Africa, the prevalence of POAG is $2.9 \%$ and it is the most common type of glaucoma in the country. ${ }^{9}$ While glaucoma has no known cure, there are treatment options by which the progression of the disease can be retarded. These methods include pharmacotherapy (oral tablets or eye drops) and surgery. Timely diagnosis and strict compliance with treatment are necessary for the successful management of glaucoma. ${ }^{10}$ Patients' knowledge and attitudes towards glaucoma are also important factors in disease management.

There has not been any research into the knowledge, attitudes and self-care practices of patients with glaucoma in South Africa. Identifying gaps in these areas will be useful in providing a basis for the development of healthcare education programmes to increase the knowledge and awareness about glaucoma, thereby improving the management of the disease. Therefore, the aim of this study was to assess the knowledge, attitudes and self-care practices of patients with glaucoma. 


\section{Research design and methods Study design}

This was an observational, analytic, cross-sectional study.

\section{Setting}

The study was conducted in the Ophthalmology Outpatient Department at Ngwelezana Hospital in the uThungulu Health District in KwaZulu-Natal. Ngwelezana Hospital is a 554-bed hospital that provides district, regional and tertiary services to an estimated 2.3 million catchment population. A private ophthalmology practice located in the same district was added as a secondary study site to ensure that the required sample size was obtained in the estimated time frame.

\section{Study population and sampling strategy}

The study population included adult patients who had been diagnosed with glaucoma for at least six months. The sample size was 384, based on the assumption that $50 \%$ of the population would have good knowledge and adequate attitudes and self-care practice for glaucoma. The following equation ${ }^{11}$ was used:

$n=\frac{Z \alpha^{2} \times p(1-p)}{d^{2}}$

[Eqn 1]

where $n=$ minimum number of respondents required; $Z \alpha=$ standard normal deviate $=1.96=($ confidence interval $[\mathrm{CI}]$ : $95 \%$ level of significance); $p=$ the $p$-value, which is the expected prevalence or proportion; and $d=$ precision margin of error (0.05).

Participants were recruited over a period of six months using convenience sampling. This non-probability sampling method was used firstly because of its advantages, which span from easy recruitment of readily available participants to good response rates, ${ }^{12}$ but also primarily because the number of patients with glaucoma who attend the Ophthalmology Outpatient Department of Ngwelezana Hospital is unknown.

\section{Data collection}

A structured close-ended questionnaire was used to interview patients. The questionnaire was modified from other studies $^{13,14}$ to suit the study population. In addition to basic sociodemographic variables, the questionnaire comprised 11 questions that assessed the patients' knowledge about the causes of glaucoma and eye drop usage. There were six statements that assessed patients' attitudes about having glaucoma and 15 questions on self-care practices that assessed compliance with treatment and storage of eye drops. As a result of the length of the questionnaire, it could not be attached to this manuscript. However, the questionnaire is available from the authors. Duration of glaucoma diagnosis was extracted from the patient's clinical record.

\section{Data analysis}

Data from the questionnaires were entered into Microsoft Excel version 2010 and Statistical Package for Social Sciences (SPSS) version 25.0 for statistical analysis. Level of education was classified as none (no formal education), primary education (Grades R-7), secondary education (Grades 8-12) and tertiary education (colleges, technical schools and universities). Patients with tertiary education were grouped with patients with secondary education during data analysis because of the small number of patients with tertiary education. Duration of glaucoma was measured as the period between the date the patient was diagnosed with glaucoma and the date of enrolment in the study and was assessed as a continuous variable.

A score of one point was awarded for each correct response in the knowledge section. Knowledge was scored out of 8 and categorised as good knowledge ( $\geq 5$ out of 8 ) or poor knowledge ( $<5$ out of 8 ). The denominator for the calculation of self-care practices scores differed according to the patients' category of self-care practice. The following categories were identified: (1) patients who took tablets and self-instilled their eye drops; (2) patients who took tablets but did not selfinstil their eye drops; (3) patients who did not take tablets but self-instilled their eye drops; and (4) patients who did not take tablets and did not self-instil their eye drops. All patients were grouped according to their self-care practice (good or poor) regardless of which category of self-care practice they were in.

Odds ratios (OR) with 95\% CI were calculated using Pearson's chi-square tests to measure associations between categorical variables. Multivariate logistic regression was conducted to assess for associations between sociodemographic variables, knowledge and self-care practice. A $p$-value less than 0.05 was considered significant.

\section{Ethical consideration}

Permission was obtained from the Chief Executive Officer of Ngwelezana Hospital, the KwaZulu-Natal Provincial Health Research and Ethics Committee and the ophthalmologist at the private practice. The study was approved by the Biomedical Research Ethics Committee, University of KwaZulu-Natal (BE430/16). The study was conducted in accordance with the World Medical Association Declaration of Helsinki on the ethical principles for medical research involving human subjects. All the approved ethical protocols such as informed consent, confidentiality and respect were followed during the study.

\section{Results}

\section{Sociodemographic characteristics of patients}

A total of 384 patients completed the questionnaire, of which $222(57.8 \%)$ were women (Table 1$)$. The age range of patients was between 26 and 80 years, with the majority $(n=117$, $30.5 \%$ ) of patients being between 56 and 65 years (Table 1 ). 
The median age of males and females was 60 (interquartile range [IQR] 48.5-69.5) and 59 years (IQR 49.0-66.5), respectively $(p>0.05)$. Nearly half of the patients $(n=182$, $47.4 \%$ ) had primary education as their highest level of education, while $113(29.4 \%)$ patients had no formal education. Less than a third of the patients were employed. Sixty-four $(16.7 \%)$ patients had a positive family history of glaucoma. The duration of glaucoma ranged from 6 months to 19 years.

\section{Patients' knowledge of glaucoma}

Two hundred and thirty-eight (62.0\%) patients had good knowledge of glaucoma and of these, 64 (26.9\%) were below the age of 46 years. Both the bivariate and multivariate analyses showed a statistically significant association between the patient's age and knowledge of glaucoma (Table 2). Patients older than 65 years were significantly less likely to have good knowledge of glaucoma than patients

TABLE 1: Sociodemographic characteristics of patients with glaucoma in the uThungulu Health District, 2016-2017.

\begin{tabular}{llcc}
\hline Characteristics & Variables & $\boldsymbol{n}$ & $\boldsymbol{\%}$ \\
\hline Gender & Female & 222 & 57.8 \\
Age (years) & Male & 162 & 42.2 \\
& $26-45$ & 77 & 20.1 \\
& $46-55$ & 80 & 20.8 \\
& $56-65$ & 117 & 30.5 \\
Level of education & $>65$ & 110 & 28.6 \\
& None & 113 & 29.4 \\
& Primary & 182 & 47.4 \\
& Secondary & 85 & 22.1 \\
Race & Tertiary & 4 & 1.0 \\
& Black & 375 & 97.7 \\
& Indian & 6 & 1.6 \\
Monthly income & White & 3 & 0.8 \\
& $<$ R500 & 22 & 5.7 \\
& R500-R1399 & 130 & 33.9 \\
& R1400-R5000 & 143 & 37.2 \\
& $>$ R5000 & 19 & 4.9 \\
& Did not disclose & 70 & 18.2 \\
\hline & Employed & 125 & 32.6 \\
& Pensioner & 107 & 27.9 \\
& Unemployed & 152 & 39.6 \\
\hline
\end{tabular}

aged 26-45 years (OR: 0.1, 95\% CI: 0.03-0.2, $p<0.001$ ). Gender was also associated with knowledge, with females being two times more likely to have good knowledge of glaucoma compared to males (OR: 2.0, 95\% CI: 1.2-3.3, $p=0.01$ ). Patients' level of education and employment status were not significantly associated with knowledge of glaucoma. The association between positive family history of glaucoma and good knowledge of glaucoma was significant (OR: 0.5, 95\% CI: $0.3-0.7, p<0.001)$. With increasing duration of glaucoma (per additional month), the patient's likelihood of having good knowledge increased and this association was statistically significant (OR: 1.1, 95\% CI: 1.1-1.2, $p<0.001$ ) (Table 2).

\section{Patients' attitudes towards glaucoma}

The majority of patients ( $n=368,95.8 \%$ ) had negative attitudes (frustrated and sad) towards their glaucoma diagnosis. However, 194 (50.5\%) patients felt happy about using their eye drops daily. In terms of disclosure, 333 (86.7\%) had informed other family members about their glaucoma diagnosis (Table 3).

\section{Self-care practices of patients with glaucoma}

The majority of patients $(n=344,89.6 \%)$ reported having good self-care practices, and of these $74(21.5 \%)$ were below the age of 46 years. In bivariate analysis, patients over the age of 65 years were significantly less likely to have good self-care practices compared to patients aged 26-45 years (OR: 0.2 , 95\% CI: $0.1-0.6, p=0.01$ ). Even though this

TABLE 3: Patients' responses to attitudinal questions on glaucoma in the uThungulu Health District, 2016-2017.

\begin{tabular}{llcc}
\hline Question & Response & $\boldsymbol{n}$ & $\boldsymbol{\%}$ \\
\hline How do you feel about & Frustrated & 101 & 26.3 \\
having glaucoma? & Happy & 15 & 3.9 \\
& Sad & 267 & 69.5 \\
How do you feel about & Frustrated & 169 & 44.0 \\
using eye drops daily? & Happy & 194 & 50.5 \\
& Sad & 17 & 4.4 \\
$\begin{array}{l}\text { Have you told your } \\
\text { family and relatives about }\end{array}$ & Yes & 333 & 86.7 \\
your glaucoma? & No & 51 & 13.3 \\
\hline
\end{tabular}

TABLE 2: Patient-related characteristics associated with their knowledge of glaucoma in the uThungulu Health District, $2016-2017$.

\begin{tabular}{|c|c|c|c|c|c|c|c|}
\hline Characteristics & Variables & Odds ratio & $95 \% \mathrm{Cl}$ & $p$ & Adjusted odds ratio & $95 \% \mathrm{Cl}$ & $p$ \\
\hline \multirow[t]{4}{*}{ Age } & $26-45$ & Referent & - & - & Referent & - & - \\
\hline & $46-55$ & 0.4 & $0.2-0.9$ & 0.02 & 0.4 & $0.2-0.8$ & 0.02 \\
\hline & $56-65$ & 0.5 & $0.2-1.0$ & 0.04 & 0.3 & $0.1-0.7$ & 0.01 \\
\hline & $>65$ & 0.1 & $0.1-0.2$ & $<0.001$ & 0.1 & $0.03-0.2$ & $<0.001$ \\
\hline \multirow[t]{2}{*}{ Gender } & Male & Referent & - & - & - & - & - \\
\hline & Female & 2.8 & $1.8-4.3$ & $<0.001$ & 2.0 & $1.2-3.3$ & 0.01 \\
\hline \multirow[t]{3}{*}{ Level of education } & None & Referent & - & - & - & - & - \\
\hline & Primary & 0.5 & $0.3-0.9$ & 0.01 & 0.6 & $0.3-1.1$ & 0.12 \\
\hline & Secondary & 0.8 & $0.4-1.5$ & 0.48 & 0.5 & $0.2-1.1$ & 0.10 \\
\hline \multirow[t]{3}{*}{ Employment status } & Unemployed & Referent & - & - & - & - & - \\
\hline & Employed & 0.6 & $0.4-1.0$ & 0.07 & 0.8 & $0.4-1.4$ & 0.40 \\
\hline & Pensioner & 0.4 & $0.3-0.7$ & $<0.001$ & 0.9 & $0.4-1.9$ & 0.79 \\
\hline Family history & Positive & 0.4 & $0.3-0.6$ & $<0.001$ & 0.5 & $0.3-0.7$ & $<0.001$ \\
\hline Duration & - & 1.2 & $1.1-1.3$ & $<0.001$ & 1.1 & $1.1-1.2$ & $<0.001$ \\
\hline
\end{tabular}

$\mathrm{Cl}$, confidence interval. 
TABLE 4: Patient-related characteristics associated with patients' glaucoma self-care practices in the uThungulu Health District, 2016-2017.

\begin{tabular}{|c|c|c|c|c|c|c|c|}
\hline Characteristics & Variables & Odds ratio & $95 \% \mathrm{Cl}$ & $p$ & Adjusted odds ratio & $95 \% \mathrm{Cl}$ & $p$ \\
\hline \multirow[t]{4}{*}{ Age } & $26-45$ & Referent & - & - & Referent & - & - \\
\hline & $46-55$ & 0.4 & $0.1-1.7$ & 0.23 & 0.6 & $0.1-2.7$ & 0.52 \\
\hline & $56-65$ & 0.4 & $0.1-1.6$ & 0.22 & 0.7 & $0.2-3.1$ & 0.63 \\
\hline & $>65$ & 0.2 & $0.1-0.6$ & 0.01 & 0.5 & $0.1-2.3$ & 0.36 \\
\hline \multirow[t]{2}{*}{ Gender } & Male & Referent & - & - & - & - & - \\
\hline & Female & 1.6 & $0.8-3.1$ & 0.17 & 1.7 & $0.7-3.7$ & 0.20 \\
\hline & Primary & 2.3 & $1.1-4.8$ & 0.03 & 3.8 & $1.5-9.7$ & 0.01 \\
\hline & Secondary & 2.0 & $0.8-4.7$ & 0.15 & 1.5 & $0.5-4.8$ & 0.48 \\
\hline \multirow[t]{3}{*}{ Employment status } & Unemployed & Referent & - & - & - & - & - \\
\hline & Employed & 1.5 & $0.6-3.7$ & 0.39 & 2.2 & $0.8-6.6$ & 0.14 \\
\hline & Pensioner & 0.5 & $0.2-1.1$ & 0.70 & 1.1 & $0.4-2.9$ & 0.85 \\
\hline Family history & Positive & 0.6 & $0.4-1.0$ & 0.06 & 0.8 & $0.4-1.3$ & 0.35 \\
\hline Duration & & 1.0 & $0.9-1.1$ & 0.64 & 1.1 & $0.9-1.1$ & 0.87 \\
\hline
\end{tabular}

$\mathrm{Cl}$, confidence interval.

association became insignificant in multivariate analysis ( $p=0.36)$, the likelihood of having good self-care practices was least in the oldest age group (> 65 years) (OR: 0.5, 95\% CI: 0.1-2.3). Patients with a primary level of education were significantly more likely to have good self-care practices compared to patients with no education (OR: 3.8, 95\% CI: $1.5-9.7, p=0.01)$. Patients with good knowledge of glaucoma were six times more likely to have good self-care practices (95\% CI: 2.6-14.1, $p<0.001$ ) (see Table 4).

\section{Discussion}

We found that almost two-thirds (approximately 67\%) of the patients with glaucoma had good knowledge of their condition in contrast to results from studies in Nigeria and Ghana where less than half $(<50 \%)$ of the patients had good knowledge of having glaucoma. The higher proportion of patients with good knowledge of glaucoma may be attributed to the patients in our study possibly receiving more information about glaucoma than the patients in other health settings. 15,16

Studies carried out in Hong Kong and Australia reported a significant association between increasing age and knowledge of glaucoma. ${ }^{17,18,19}$ However, in our study, we observed a reduction in the level of knowledge of patients with increasing age. A possible explanation for this finding may be that older patients forget information more easily than younger patients as a result of senile changes. Females were more knowledgeable about glaucoma than males in our study. This finding is in keeping with the available body of evidence that females are more inclined to practice health-seeking behaviours in comparison to males..$^{13,17,20,21}$ There is a therefore a need for the sensitisation and education of males about glaucoma, especially as the risk of having POAG is higher in males than females. ${ }^{5,6,22,23}$

Family disclosure of one's glaucoma diagnosis is important because genetics and family history are risk factors of glaucoma. ${ }^{24}$ It is interesting to note that the majority $(86.7 \%)$ of patients in our study had told their family members about their glaucoma diagnosis, which is consistent with literature from a Jamaican study. ${ }^{14}$ Several studies have reported that patients who had one or more family members with a diagnosis of glaucoma were more knowledgeable than those who did not have a family history of glaucoma. ${ }^{17,21,25,26}$ However, the patients in our study were less likely to have good knowledge of glaucoma if they had a positive family history. The reason for this may be that patients who have family members with glaucoma may not pay attention when they are being counselled about their condition as they feel they already have knowledge about the disease.

Regarding the impact of duration of glaucoma on knowledge, our finding is consistent with other studies. ${ }^{27,28,29}$ Patients who have had glaucoma for a longer period would have had more opportunities to participate in glaucoma health talks during their follow-up visits to the hospital and to discuss their condition with the doctor, thereby improving their knowledge of glaucoma.

The methods used to assess self-care practices in patients with glaucoma differ across studies. ${ }^{13,18,30,31}$ It is therefore difficult to compare our results with these studies. We observed that older patients were less likely to have good self-care practices than younger patients. Although this finding was not statistically significant, it is likely that older patients would be more prone to forgetting recommended doses and timing of their medications. Our finding that good knowledge of glaucoma is associated with good self-care practices is consistent with results from a study conducted in Ethiopia. ${ }^{32}$

\section{Strengths and limitations}

With the absence of previous studies in South Africa on the knowledge, attitudes and self-care practices of patients with glaucoma, the results of this study provides valuable information that can assist in the development of educational material on glaucoma for patients and their families. 
Recall bias is notable in studies that encompass some measure of self-reporting. The patients in this study were required to answer questions that entailed remembering past events and as such it is possible there might have been some inaccuracies in their recollections, especially with the older patients (e.g. where patients were asked to say how often they forget to use their glaucoma medication).

\section{Recommendations}

We recommend that more time and repeated education sessions be made available for patients with glaucoma, particularly the elderly and male patients. It is also important to involve the family members of these patients in glaucoma education and counselling to facilitate improved disease management.

\section{Conclusion}

Elderly patients do not have sufficient knowledge of glaucoma and have poor glaucoma self-care practices. Therefore, although the general population will benefit from glaucoma awareness campaigns and educational programmes, concerted efforts and focus on reaching this group of individuals and those more at risk of the disease will be highly beneficial.

\section{Acknowledgements}

The authors would like to thank Ms Bongi Ntuli for the translation of the study tool from English to IsiZulu and Dr Johann de Lange for granting them permission to utilise his ophthalmology practice as an additional study site.

\section{Competing interests}

The authors declare no competing interests with regard to the writing of this article.

\section{Authors' contributions}

A.V.A. was responsible for the project design, data collection (with the assistance of trained personnel) and writing of the manuscript. S.M. assisted in refining the research concept and study method, supervised the research process and reviewed all versions of the article. B.T. supervised data analysis.

\section{Funding information}

A.V.A. received a grant from the College of Health Sciences, University of KwaZulu-Natal, for the research.

\section{References}

1. Omoti AE, Edema OT, Akpe BA, Musa P. Cost analysis of medical versus surgical management of glaucoma in Nigeria. J Ophthalmic Vis Res. 2010:5(4):232-239. https://doi.org/10.4314/jmbr.v1i1.10650

2. Mohamed EA, Bayoumi OR, Draz SF. Impact of an educational programme on knowledge, beliefs, practices and expectations about care among adolescent glaucoma patients in Cairo. East Mediterr Health J. 2011;17(12):960-968. https:// doi.org/10.26719/2011.17.12.960
3. Quigley HA. New paradigms in the mechanisms and management of glaucoma. Eye. 2005;19(12):1241-1248. https://doi.org/10.1038/sj.eye.6701746

4. Tham YC, Li X, Wong TY, Quigley HA, Aung T, Cheng CY. Global prevalence of glaucoma and projections of glaucoma burden through 2040: A systematic review and meta-analysis. Am J Ophthalmol. 2014;121(11):2081-2090. https://doi. org/10.1016/j.ophtha.2014.11.030

5. Quigley HA, Broman AT. The number of people with glaucoma worldwide in 2010 and 2020. Br J Ophthalmol. 2006;90(3):262-267. https://doi. org/10.1136/bjo.2005.081224

6. Rotchford AP, Johnson GJ. Glaucoma in Zulus: A population-based cross-sectional survey in a rural district in South Africa. Arch Ophthalmol. 2002;120(4):471-478. https://doi.org/10.1001/archopht.120.4.471

7. Rotchford AP, Kirwan JF, Muller MA, Johnson GJ, Roux P. Temba glaucoma study: A population-based cross-sectional survey in urban South Africa. Ophthalmology. 2003;110(2):376-382. https://doi.org/10.1016/s0161-6420(02) Ophthalmol

8. Cook CD, Knight SE, Crofton-Briggs I. Prevalence and causes of low vision and blindness in northern KwaZulu. S Afr Med J. 1993;83(8):590-593.

9. De-Gaulle VF, Dako-Gyeke P. Glaucoma awareness, knowledge, perception of risk and eye screening behaviour among residents of Abokobi, Ghana. BMC Ophthalmol. 2016;16:204. https://doi.org/10.1186/s12886-016-0376-0

10. Kyari F, Abdull MM, Bastawrous A, Gilbert CE, Faal H. Epidemiology of glaucoma in sub-Saharan Africa: Prevalence, incidence and risk factors. Middle East Afr J Ophthalmol. 2013;20(2):111-125. https://doi.org/10.4103/ 0974-9233.110605

11. Bohn RL, Gurwitz JH, Yeomans SM, et al. Which patients are treated for glaucoma? An observational analysis. J Glaucoma. 2000;9(1):38-44. https://doi.org/ An observational analysis. J Glauco

12. Lamoureux EL, Saw SM, Thumboo J, et al. The impact of corrected and uncorrected refractive error on visual functioning: The Singapore Malay Eye Study. Invest Ophthalmol Vis Sci. 2009;50(6):2614-2620. https://doi.org/ 10.1167/iovs.08-2164

13. Mabaso RG, Oduntan OA. Knowledge and practices related to diabetes mellitus among adults with diabetes in the Mopani District, Limpopo Province, South Africa. Afr Vision Eye Health. 2016;75(1):1-6. https://doi.org/10.4102/aveh.v75i1.324

14. Mohindroo C, Ichhpujani $P$, Kumar S. How 'drug aware' are our glaucoma patients? J Curr Glaucoma Pract. 2015;9(2):33-37. https://doi.org/10.5005/jpjournals-10008-1181

15. Grant A. A cross-sectional, descriptive study to measure: Knowledge, attitudinal and behavioural (social practices) effects which a positive diagnosis of glaucoma may have on affected patients of the Eye Department of the FISH Medical Clinic, Gordon Town Road, St. Andrew, Jamaica. Jamaica: University of West Indies; 2010.

16. Sathyamangalam R, Paul P, George R, et al. Determinants of glaucoma awareness and knowledge in urban Chennai. Indian J Ophthalmol. 2009;57(5):355-360. https://doi.org/10.4103/0301-4738.55073

17. Onunkwor C, Monareng L. Glaucoma knowledge of patients in Abuja, Nigeria. Afr J Nurs Midwifery. 2012;14(1):3-15.

18. Nkum G, Lartey S, Frimpong C, Micah F, Nkum B. Awareness and knowledge of glaucoma among adult patients at the eye clinic of a teaching hospital. Ghana Med J. 2015;49(3):195-199. https://doi.org/10.4314/gmj.v49i3.11

19. Landers JA, Goldberg I, Graham SL. Factors affecting awareness and knowledge of glaucoma among patients presenting to an urban emergency department. Clin Exp Ophthalmol. 2002;30(2):104-109. https://doi.org/10.1046/j.1442 6404.2002.00493.x

20. Livingston PM, McCarty CA, Taylor HR. Knowledge, attitudes, and self care practices associated with age related eye disease in Australia. $\mathrm{Br} J$ Ophthalmol. 1998;82(7):780-785. https://doi.org/10.1136/bjo.82.7.780

21. Lau JTF, Lee V, Fan D, Lau M, Michon J. Knowledge about cataract, glaucoma, and age related macular degeneration in the Hong Kong Chinese population. $\mathrm{Br} J$ Ophthalmol. 2002;86(10):1080-1084. https://doi.org/10.1136/bjo.86. 10.1080

22. Livingston PM, Lee SE, De Paola C, Carson CA, Guest CS, Taylor HR. Knowledge of glaucoma, and its relationship to self-care practices, in a population sample. Clin Exp Ophthalmol. 1995;23(1):37-41. https://doi.org/10.1111/j.1442-9071.1995. tb01643.x

23. Ogbonnaya CE, Ogbonnaya LU, Okoye O, Kizor-Akaraiwe N. Glaucoma awareness and knowledge, and attitude to screening, in a rural community in Ebonyi State, Nigeria. Open J Ophthalmol. 2016;6(2):119-127. https://doi.org/10.4236/ojoph. 2016.62017

24. Kyari F, Entekume G, Rabiu M, et al. A Population-based survey of the prevalence and types of glaucoma in Nigeria: Results from the Nigeria National Blindness and Visual Impairment Survey. BMC Ophthalmol. 2015;15:176. https://doi. org/10.1186/s12886-015-0160-6

25. Salmon JF, Mermoud A, Ivey A, Swanevelder SA, Hoffman M. The prevalence of primary angle closure glaucoma and open angle glaucoma in Mamre, Western Cape, South Africa. Arch Ophthalmol. 1993;111(9):1263-1269. https://doi. org/10.1001/archopht.1993.01090090115029

26. Australia Government. NHMRC guidelines for the screening, prognosis, diagnosis, management and prevention of glaucoma [homepage on the Internet]. Australia Government; 2010 [cited 2016 Apr 25]. Available from: https://www.nhmrc.gov. au/guidelines-publications/cp113-cp113b 
27. Rewri P, Kakkar M. Awareness, knowledge, and practice: A survey of glaucoma in north Indian rural residents. Indian J Ophthalmol. 2014;62(4):482-486. https://doi.org/10.4103/0301-4738.132105

28. Hoevenaars J, Schouten J, van den Borne B, Beckers H, Webers C. Knowledge base and preferred methods of obtaining knowledge of glaucoma patients. Eur Ophthalmol. 2005;15(1):32-40. https://doi.org/10.1177/112067210501500106

29. Hoevenaars JGMM, Schouten JSAG, van den Borne B, Beckers HJM, Webers CAB. Socioeconomic differences in glaucoma patients' knowledge, need for information and expectations of treatments. Acta Ophthalmol Scand. 2006;84(1):84-91. https://doi.org/10.1111/1.1600-0420.2005.00587.x
30. Danesh-Meyer HV, Deva NC, Slight C, et al. What do people with glaucoma know about their condition? A comparative cross-sectional incidence and prevalence survey. Clin Exp Ophthalmol. 2008;36(1):13-18. https://doi.org/10.1111/j.1442survey. Clin Exp Opht1.2007.01624.x

31. Tsai T, Robin AL, Smith JP, 3rd. An evaluation of how glaucoma patients use topical medications: A pilot study. Trans Am Ophthalmol Soc. 2007;105: 29-35.

32. Elfride FS, Philip S, Kishore SR. Knowledge, attitude, practice and quality of life assessment in glaucoma - A cross sectional survey. Int J Med Res Rev. 2016;4(12):2199-2204. https://doi.org/10.17511/ijmrr.2016.i12.20 